\title{
Extracting pairwise comparisons data from best-worst scaling surveys by logical inference
}

Technical Report No. 223a

Ulf A. Hamster

orcid: 0000-0002-0440-4868

March 14, 2021

\begin{abstract}
Best-Worst Scaling (BWS) based user interfaces allow efficient evaluation of many items through a low number of user actions. In addition to the explicit selection of the best and the worst item, more paired comparisons can be extracted from a BWS set using the unselected items. In this report, we explore how to extract further paired comparisons by logical inference between two BWS sets. Logically inferred pairs can be interpreted as implicit choices and used to validate the consistency of explicitly extracted pairs or to enrich datasets or resp. reduce data collection costs.
\end{abstract}

\section{Introduction}

Best-Worst Scaling FL92 have been used to label data for word sense disambiguation [Jur13 and sentiment analysis [KM17].

\section{Extract pairs from one BWS set}

A Best-Worst Scaling (BWS) user interface (UI) displays a set of $M \geq 3$ items (BWS set), and the user selects the best and worst item. The UI returns a $M$-tuple combinatorial state with the possible item states BEST, WORST, and NOT (i.e. unselected) from which

$$
\frac{M(M-1)}{2}-\frac{K(K-1)}{2}=\frac{M(M-1)}{2}-\frac{(M-2)(M-3)}{2}=2 M-3
$$

pairs can be extracted. The Fig. 1 exhibits the case for $M=4$ items.

\section{The yield-effort ratio to gather ranked data}

The effort using an UI can be measured in terms of user actions (e.g. clicks, drag and drop, voice commands). An UI for direct pairwise comparison between two choices $C_{i}$ and $C_{j}$ would require $A=1+x$ actions: (1) Select the BEST item, and optionally (x) push the submit button or other actions. The pairwise comparison UI follows a constant return to scale (CRS) production function [econ.] with the property $f(\beta A)=\beta f(A)$.

The BWS UI require $A=2+x$ actions for evaluation: (1) Select the BEST, (2) select the WORST, and optionally (x) push the submit button or other actions. In case of BWS the number of pairs $f$ also depends the number of items $M$ displayed. For a given 

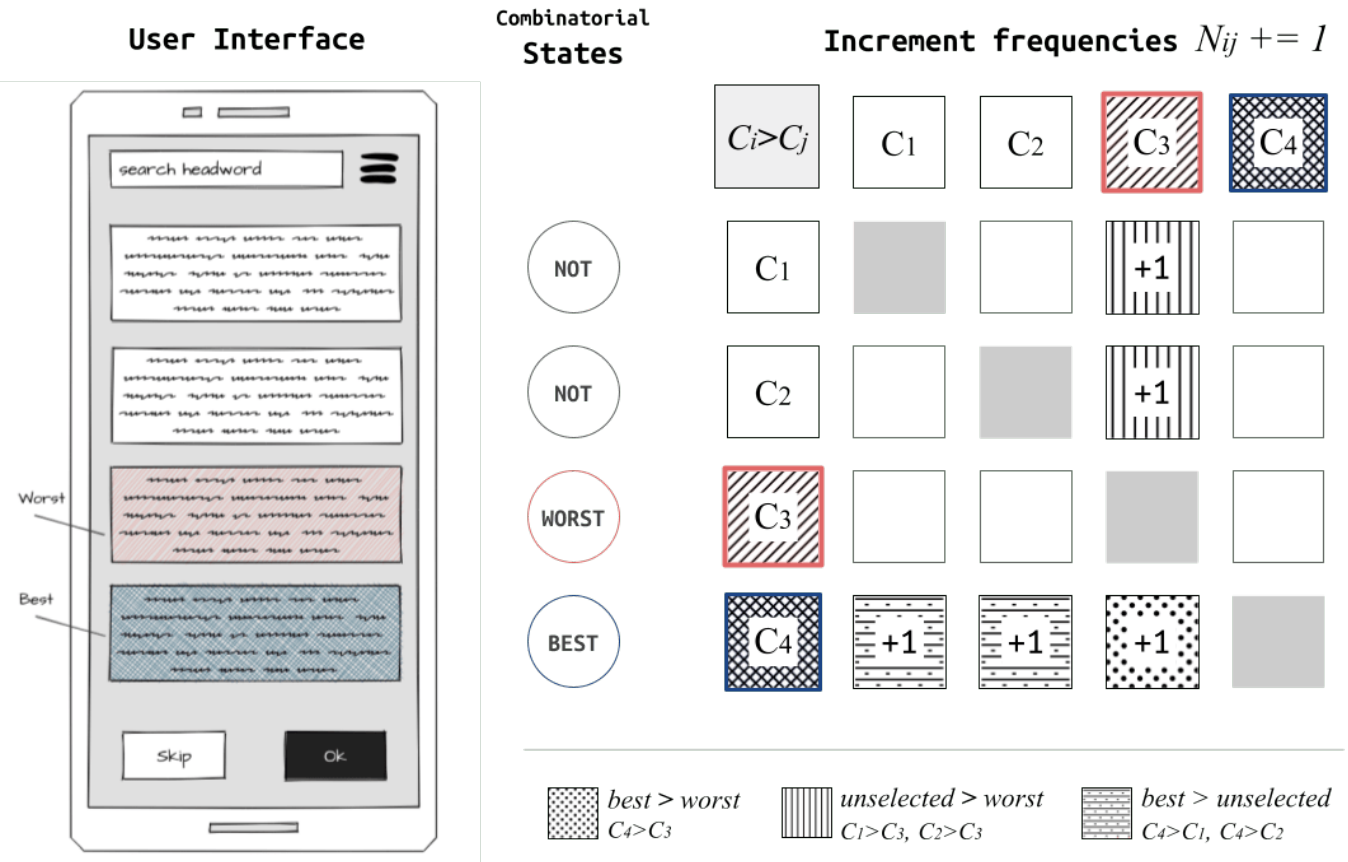

Figure 1: User interface for BWS surveys in mobile portrait mode (left), the resulting combinatorial states (center), and counting extracted pairs in a sparse matrix (right). The illustration shows an evaluated $B W S$ set $\left(C_{1}, C_{2}, C_{3}, C_{4}\right)$ with $C_{4}$ selected best and $C_{3}$ as worst item.

number of actions $A=2+0$, a BWS UI yields $f(A=2, M)=2 M-3$ pairs (Eq. 1). $f$ is an increasing returns to scale (IRS) production function [econ.] with the property $f(\beta A, \beta M)>\beta f(A, M) \forall \beta>1$ (Tab. 1).

\begin{tabular}{c|cc} 
& $f(\beta A, \beta M)$ & $\beta f(A, M)$ \\
\hline \hline$\beta=1$ & 3 & 3 \\
$\beta=2$ & $2 \cdot 9=18$ & 6 \\
$\beta=3$ & $3 \cdot 15=45$ & 9 \\
\hline
\end{tabular}

Table 1: Increasing the number of items $M$ by factor $\beta$ causes the increasing returns to scale (IRS) effect.

The yield-effort percentage ratio $r(M)$ with $M \geq 3$ can be used to compare the efficiency of using a BWS UI over a pairwise comparison UI:

$$
r(M)=\frac{\max (0,2 M-3)}{2}-1=\max (0, M-1.5)-1
$$

When comparing the the yield-effort ratio $r(M)$ for different number of items $M$ in a BWS set (Tab. 2), then it would be tempting to set $M \rightarrow \infty$ (i.e. display as many items as possible in the BWS UI). However, the number of items $M$ that can be processed by an human is limited [Mil56] depending on "conditions in which [information] are held in the focus of attention at one time" Cow01, p. 114]. Possible factors might be a) the users' general abilities, b) the items' content and its newness and complexity to the user, c) the decision criteria and its newness and complexity to the user, and d) the cognitive exhaustion of the user over the course of the experiment. Cow01 offers a good overview about working memory capacity. 


\begin{tabular}{cc}
$M$ & $r(M)$ \\
\hline \hline 2 & $-50 \%$ \\
3 & $+50 \%$ \\
4 & $+150 \%$ \\
5 & $+250 \%$ \\
6 & $+350 \%$ \\
\hline
\end{tabular}

Table 2: Yield-effort ratio $r(M)$ of BWS UIs for different number of items $M$ compared to a pairwise comparison UI.

\section{Logical Inference from two BWS sets}

A second way to extract paired comparisons is by logical inference from overlapping BWS sets. A newly evaluated BWS set is assessed against a database of previously processed BWS sets. Fig. 2 illustrate the 9 possible ways how 2 BWS sets can overlap, and how many

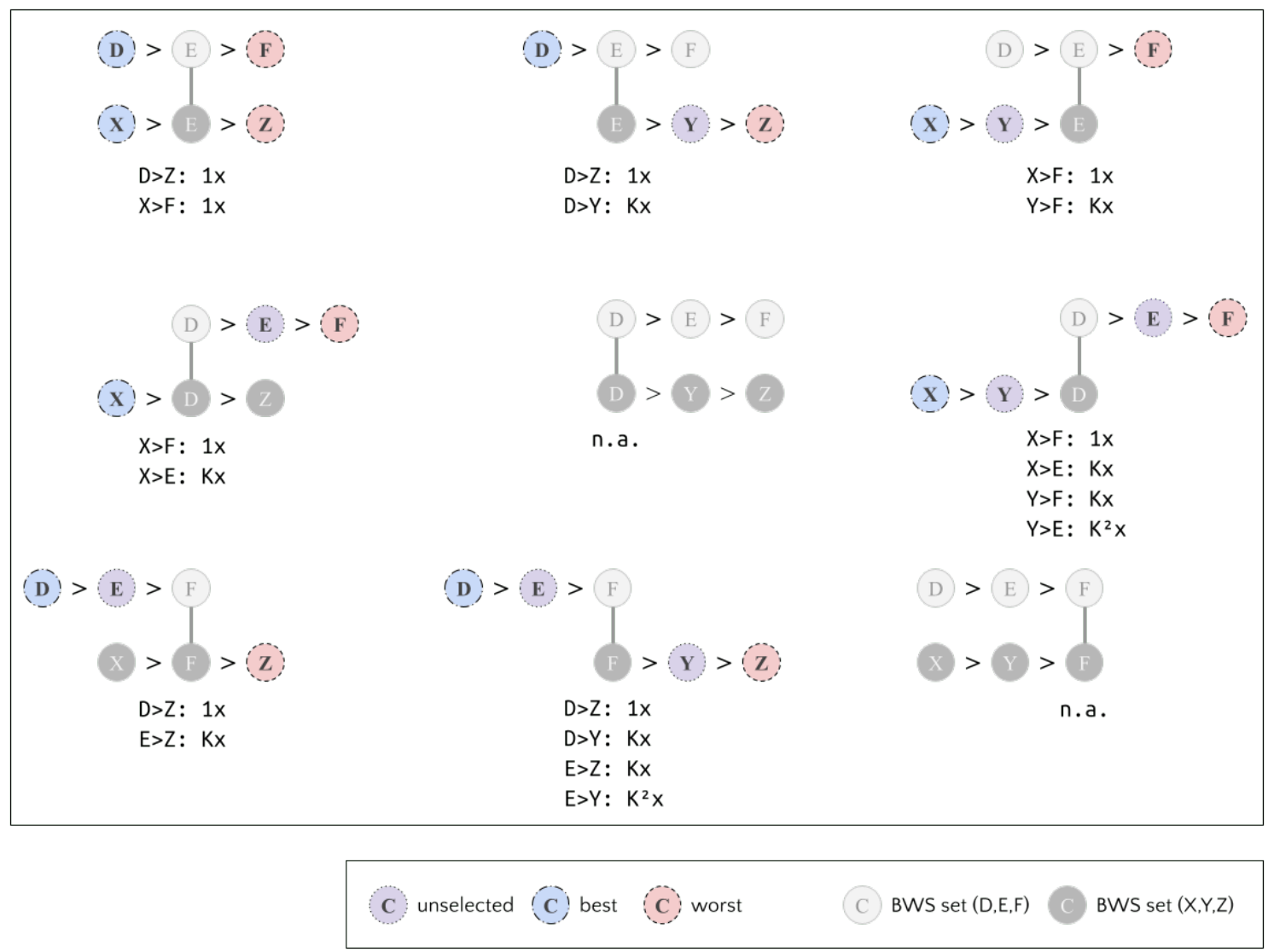

Figure 2: The nine cases to logical infer pairwise comparisons from two $B W S$ sets $(D, E, F)$ and $(X, Y, Z)$. The number of logical inferred pairs is counted in terms of $K=M-2$ units.

paired comparisons can be inferred for each combination. In total $8\left(1+K+\frac{K^{2}}{4}\right)$ logical inferred pairs, with $K=M-2$. When comparing a new BWS set against a database of $H=1$ BWS sets, the expected number of pairs $\mathbb{E}[M]$ for $M \geq 3$ is

$$
\mathbb{E}[M]=\frac{8}{9} \cdot\left(M-1+\frac{(M-2)^{2}}{4}\right)
$$

what is comparable to the number of pairs that can be extracted directly from BWS set (Tab. 3). The total number of logical inferred pairs can rise to $H \cdot \mathbb{E}[M]$ depending on the database size $H$. Given the number of BWS sets $G$ that are about to be evaluated, the 


\begin{tabular}{ccc}
\hline$M$ & $\begin{array}{c}\text { Directly } \\
\text { extracted } \\
2 M-3\end{array}$ & $\begin{array}{c}\text { Logical } \\
\text { inferred } \\
\mathbb{E}[M]\end{array}$ \\
\hline \hline 3 & 3 & 2.0 \\
4 & 5 & $3 . \overline{5}$ \\
5 & 7 & $5 . \overline{5}$ \\
6 & 9 & 8.0 \\
7 & 11 & $10 . \overline{8}$ \\
\hline
\end{tabular}

Table 3: Number of pairs directly extracted from 1 BWS set, and the expected number of pairs of $1 B W S$ set that can be logical inferred from a database of $H=1$ BWS sets.

algorithm has quadratic $\mathcal{O}(G \cdot H)$ complexity, and thus, it may be beneficial to restrict the number of BWS sets $H$ of such a database.

\section{$5 \quad$ Sampling Strategy}

In order to ensure overlapping BWS sets, a pool of examples can be arranged into $G$ sets of $M$ items as illustrated in Fig. 3. First, every $(l \cdot(M-1)+1)$-th example is assigned to two consecutive BWS sets, so that $1 /(M-1)$ of examples are evaluated two times. Second, the remaining $(M-2) /(M-1)$ non-overlapping examples are assigned into further BWS sets (See lines in Fig. 3).

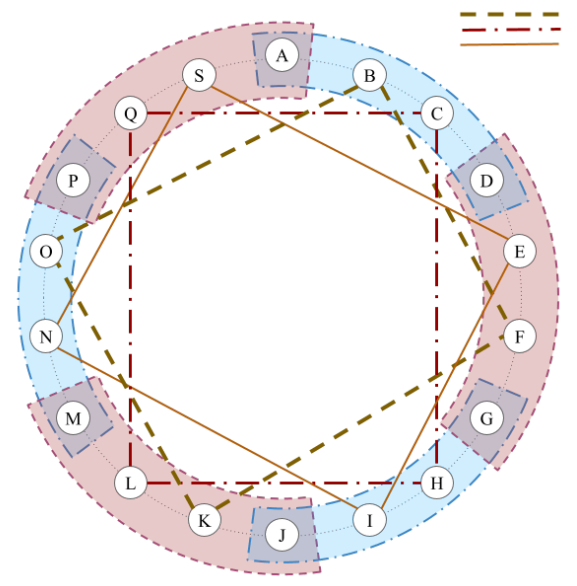

Figure 3: Arrange a pool of examples $[A, B, C, \ldots]$ so that $B W S$ sets overlap. Then connect non-overlapping items to further $B W S$ sets so that very item is part of at least two $B W S$ sets.

\section{$6 \quad$ Usage Example}

The logical inference rules are implemented in the PyPi package bwsample (v0.5.4) and applied to the sentiment dataset ${ }^{1}$ from $\left[\right.$ KM17 who computed the scores ${ }^{2}$ according to Orm09.

As expected the Tab. 4 shows that (b) scores based on directly extracted pairs and (d) reported scores in [KM17] are highly rank-correlated $\left(\rho_{b, d}=.9917\right)$. The scores based on

\footnotetext{
${ }^{1}$ see "BWS annotations", http://www.saifmohammad.com/WebDocs/bwsvsrs/BWS annotations.csv

${ }^{2}$ see "Scores obtained by BWS", http://www.saifmohammad.com/WebDocs/bwsvsrs/BWS-scores.txt
} 
logical inferred pairs (c) are slightly less rank-correlated to (b) scores based on directly extracted pairs $\left(\rho_{b, c}=.9767\right)$, and $(\mathrm{d})$ the reported scores $\left(\rho_{c, d}=.9656\right)$. A possible explanation are contradicting user answers due to inconsistent decision-making (e.g. user chooses $C_{1}>C_{2}$ and later $C_{2}>C_{3}$ and $C_{3}>C_{1}$ ) or different opinions between two or more user groups.

\begin{tabular}{l|cc|c|r}
\hline & (b) & (c) & (d) & $M$ \\
\hline \hline (a) only best \& worst & .9365 & .9312 & .9295 & 59,815 \\
(b) directly extracted & - & .9767 & .9917 & 299,075 \\
(c) logical inferred & - & - & .9656 & $37,562,516$ \\
\hline (d) scores from KM17 & - & - & - & - \\
\hline
\end{tabular}

Table 4: Spearman rank correlations between scores based on (a) only explicit best 83 worst choices, (b) directly extracted pairs from each BWS set, (c) logical inferred pairs, and d) scores reported in [KM17].

In a second step the sentiment dataset was reduced from 59900 BWS sets to 6291 unique BWS sets, i.e. each combination of items occurred only once, and the first answer by any user was selected from the original dataset. Tab. 5 shows that the rank-correlation of (b) and (c) to $(\mathrm{d})$ the reported scores $\left(\rho_{b, d}=.9550, \rho_{c, d}=.9314\right)$ declined both compared to Tab. 4. However, the rank-correlation between (b) and (c) remained about the same $\left(\rho_{b, c}=.9767\right.$ in Tab. 4 versus $\rho_{b, c}=.9799$ in Tab. 5) what might be an indication that (b) scores from directly extracted pairs are still consistent with smaller datasets.

\begin{tabular}{l|cc|c|r}
\hline & (b) & (c) & (d) & $M$ \\
\hline \hline (a) only best \& worst & .9136 & .9039 & .8708 & 6,280 \\
(b) directly extracted & - & .9799 & .9550 & 31,400 \\
(c) logical inferred & - & - & .9314 & 398,986 \\
\hline (d) scores from KM17 & - & - & - & - \\
\hline
\end{tabular}

Table 5: Scores for (a), (b), and (c) were computed based on 6291 BWS sets while (d) uses 59900 BWS sets.

\section{Conclusion}

Logical inference rules to post-process BWS data might be useful in situation when the number of unlabelled examples is far larger than the number of human annotators.

\section{References}

[Mil56] George A. Miller. "The magical number seven, plus or minus two: some limits on our capacity for processing information". In: Psychological Review 63.2 (1956), pp. 81-97. DOI: $10.1037 / \mathrm{h} 0043158$.

[FL92] Adam Finn and Jordan J. Louviere. "Determining the Appropriate Response to Evidence of Public Concern: The Case of Food Safety". In: Journal of Public Policy \& Marketing 11.2 (Sept. 1992). Publisher: SAGE Publications Inc, pp. 1225. ISSN: 0743-9156. DOI: 10.1177/074391569201100202.

[Cow01] Nelson Cowan. "The magical number 4 in short-term memory: A reconsideration of mental storage capacity". In: Behavioral and Brain Sciences 24.1 (Feb. 2001), pp. 87-114. DOI: $10.1017 /$ S0140525X01003922. 
[Orm09] Bryan Orme. "MaxDiff Analysis: Simple Counting, Individual-Level Logit, and HB". en. 2009. URL:https://sawtoothsof tware.com/uploads/sawtoothsoftware/ originals/f89a6537-1cae-4fb5-afad-9d325c2a3143.pdf

[Jur13] David Jurgens. "Embracing Ambiguity: A Comparison of Annotation Methodologies for Crowdsourcing Word Sense Labels". In: Proceedings of the 2013 Conference of the North American Chapter of the Association for Computational Linguistics: Human Language Technologies. Atlanta, Georgia: Association for Computational Linguistics, June 2013, pp. 556-562. URL: https://www.aclweb org/anthology/N13-1062 (visited on 03/09/2021).

[KM17] Svetlana Kiritchenko and Saif Mohammad. "Best-Worst Scaling More Reliable than Rating Scales: A Case Study on Sentiment Intensity Annotation". In: Proceedings of the 55th Annual Meeting of the Association for Computational Linguistics (Volume 2: Short Papers). Vancouver, Canada: Association for Computational Linguistics, July 2017, pp. 465-470. DOI: 10.18653/v1/P17-2074.

\section{Nomenclature}

\begin{tabular}{ccl}
\hline symbol & type & description \\
\hline \hline$M$ & $\mathbb{N}$ & number of items displayed to the users in an BWS set \\
$K$ & $\mathbb{N}$ & number of unselected items, $K=M-2$ \\
$C_{i}$ & - & name of the $i$-th item (or resp. $i$-th choice) \\
$i, j$ & $\mathbb{N}_{>0}$ & position index of the items $C_{i}$ and $C_{j}$ \\
$N_{i j}$ & $\mathbb{N}_{\geq 0}$ & number of times (count, frequency) users choose $C_{i}>C_{j}$ \\
\hline$A$ & $\mathbb{N}_{>0}$ & number of user actions \\
$f()$. & $\mathbb{N}_{\geq 0}$ & number of pairs produced by using a specific user interface (UI) \\
$r(M)$ & $\mathbb{R}$ & yield-effort ratio of using a BWS UI over a pairwise comparison UI \\
\hline$G$ & $\mathbb{N}$ & number of BWS sets to be evaluated, each with $M$ items \\
$H$ & $\mathbb{N}$ & number of BWS sets in a database for logical inference \\
$\mathbb{E}[M]$ & $\mathbb{R}$ & expected number of pairs extracted by logical inference \\
\hline
\end{tabular}

Table 6: Used symbols and notations used in this report. 